\title{
Nachbereitung: Arbeiten im Anschluss an die Exkursion
}

Um einen Bogen vom Anfang, d. h. der Erstellung der Referate und/oder Hausarbeiten zu einem Einzelthema über die Erfahrungen während der Exkursion bis zum weiteren Studium zu spannen, empfiehlt es sich - je nachdem, was von den Dozent*innen eingefordert wird - am Ende oder nach der Exkursion noch einmal die gewonnenen Erkenntnisse zu bündeln. Dies kann dadurch geschehen, dass die vorliegende Hausarbeit um einen empirischen Teil ergänzt wird. Leitend sollte hier die Frage sein, was durch die Exkursion an zusätzlichen, d. h. über die Hausarbeit hinausgehenden Erkenntnissen gewonnen werden konnte. Was wurde während der Exkursion an konkreten Beispielen u. a. durch Beobachtungsaufträge, Kartierungen o. ä. erfasst? Wie wurde dies diskutiert und wie steht es mit den vorhandenen schriftlichen Ausführungen im Zusammenhang? Welches zusätzliche Wissen haben vor allem die Expert*innen vermittelt? In welcher Hinsicht kann der Forschungsstand zum Thema durch diese Interviews noch empirisch angereichert und fundiert werden? Solche Formen der erkenntniszentrierten Ergebnisdarstellung ermöglichen im Vergleich zu überkommenen Formen rein deskriptiver Protokolle eine konzeptionell rückgebundene Reflexion. Denn so kann auch noch einmal dem Grundansatz, etablierte Sichtweisen zu hinterfragen und die Vielfalt von Lebenswelten und Weltbildern vor Ort zu reflektieren und das theoretische Wissen um praktische Erfahrungen zu erweitern, Rechnung getragen werden. Darüber hinaus empfiehlt es sich, mit einem gewissen Abstand Revue passieren zu lassen und zu bewerten, was nicht nur inhaltlich, sondern auch didaktisch und organisatorisch gut gelaufen ist und was hätte besser gemacht werden können. So kann das „lehrende Lernen“ weiter gefördert werden (Dickel 2006, S. 198; Glasze 2009, S. 180). 